\title{
Design and performance of a high resolution, low latency stripline beam position monitor system
}

\author{
R. J. Apsimon, ${ }^{*}$ D. R. Bett, ${ }^{\dagger}$ N. Blaskovic Kraljevic, P. N. Burrows, G. B. Christian, ${ }^{\ddagger}$ \\ C. I. Clarke, ${ }^{\S}$ B. D. Constance, H. Dabiri Khah, M. R. Davis, C. Perry, \\ J. Resta López, and C. J. Swinson \\ John Adams Institute for Accelerator Science at University of Oxford, Denys Wilkinson Building, \\ Keble Road, Oxford OX1 3RH, United Kingdom \\ (Received 1 October 2014; published 19 March 2015)
}

\begin{abstract}
A high-resolution, low-latency beam position monitor (BPM) system has been developed for use in particle accelerators and beam lines that operate with trains of particle bunches with bunch separations as low as several tens of nanoseconds, such as future linear electron-positron colliders and free-electron lasers. The system was tested with electron beams in the extraction line of the Accelerator Test Facility at the High Energy Accelerator Research Organization (KEK) in Japan. It consists of three stripline BPMs instrumented with analogue signal-processing electronics and a custom digitizer for logging the data. The design of the analogue processor units is presented in detail, along with measurements of the system performance. The processor latency is $15.6 \pm 0.1 \mathrm{~ns}$. A single-pass beam position resolution of $291 \pm 10 \mathrm{~nm}$ has been achieved, using a beam with a bunch charge of approximately $1 \mathrm{nC}$.
\end{abstract}

DOI: 10.1103/PhysRevSTAB.18.032803

PACS numbers: 29.27.Fh, 41.85.Qg, 41.75.Ht, 29.20.db

\section{INTRODUCTION}

A number of in-construction and proposed future particle accelerator designs feature trains of particle bunches with bunch-separation intervals in the ranges of nanoseconds to tens or hundreds of nanoseconds. For example, the International Linear Collider (ILC) design [1] calls for bunch trains comprising thousands of bunches separated in time by around $500 \mathrm{~ns}$ with a train repetition frequency of $5 \mathrm{~Hz}$; the Compact Linear Collider (CLIC) design [2] specifies bunch trains comprising several hundred bunches separated in time by around $0.5 \mathrm{~ns}$, with a train repetition frequency of $50 \mathrm{~Hz}$. Free-electron lasers based on similar accelerating technologies as ILC and CLIC will have similar bunch-train time structures, such as the European XFEL [3], which will have a minimum bunch spacing of $200 \mathrm{~ns}$ and a repetition rate of $10 \mathrm{~Hz}$. Beam control at such facilities calls for beam position monitors (BPMs) that can resolve bunches on an intratrain (ideally bunch-by-bunch)

\footnotetext{
*Present address: Cockcroft Institute, University of Lancaster, UK.

Present address: CERN, Geneva, Switzerland.

g.christian1@physics.ox.ac.uk

${ }^{\S}$ Present address: SLAC National Accelerator Laboratory, Stanford, CA, USA.

"Present address: Cockcroft Institute, University of Liverpool, UK. USA.

Present address: Brookhaven National Laboratory, Upton, NY,

Published by the American Physical Society under the terms of the Creative Commons Attribution 3.0 License. Further distribution of this work must maintain attribution to the author(s) and the published article's title, journal citation, and DOI.
}

time scale, with submicron position resolution in singlepass mode. The design of such a BPM system is presented here.

The system was developed by the Feedback on Nanosecond Timescales (FONT) group [4] and it was deployed, commissioned and tested at the Accelerator Test Facility (ATF) [5] at KEK. The ATF (Fig. 1) is a test accelerator for the generation of very low emittance electron beams as required for next-generation electron-positron linear colliders. In 2008 a new beam extraction line ("ATF2" [6]) was installed at ATF. This includes a beam line with a system of magnetic focusing and steering elements that forms a low-energy prototype of a design $[7,8]$ for the final-focus system of ILC or CLIC. The goals of the ATF2 collaboration [9] are to produce an electron beam spot with a $37 \mathrm{~nm}$ vertical size, and eventually to stabilize the vertical beam position at the focal point at the nanometer level.

To this end, the FONT group has developed a beam position and angle feedback system ("FONT5") [10] for stabilizing the vertical beam position in the upstream

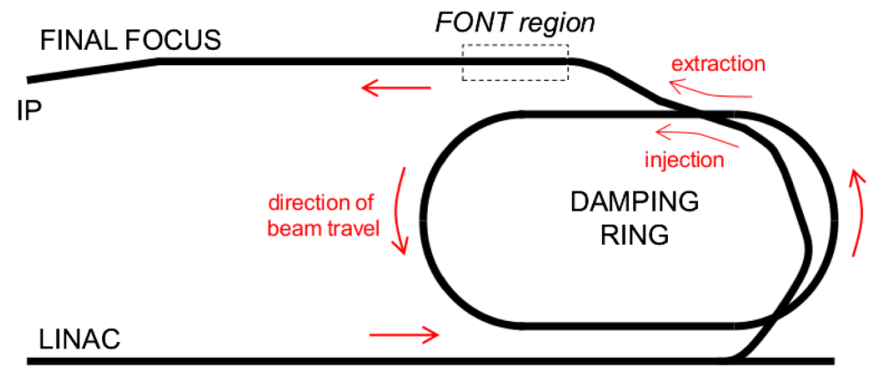

FIG. 1. Layout of the ATF, showing the locations of the ATF2 extraction line and the FONT5 system. 


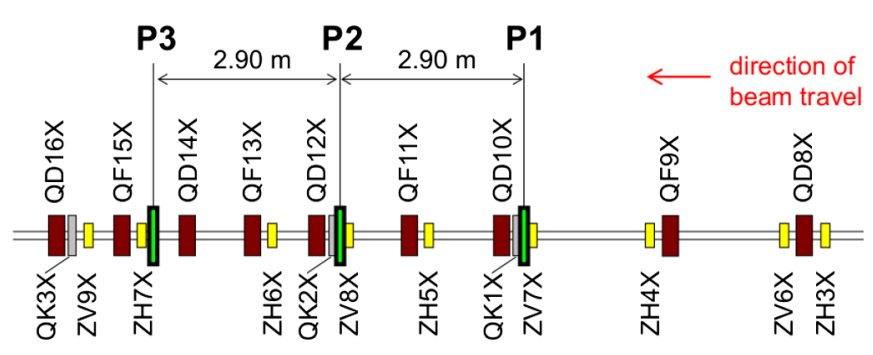

FIG. 2. Layout of the FONT5 BPMs (P1, P2 and P3) in the ATF2 extraction line; quadrupole ("Q") and dipole corrector (“Z”) magnets are indicated.

section of the ATF2 beam line (Fig. 1); the layout of the BPMs is shown in more detail in Fig. 2. The design goal for the FONT5 system is to stabilize the vertical beam position to the $1 \mu \mathrm{m}$ level at the entrance to the finalfocus system. This requires BPMs capable of resolving bunches separated in time by around $100 \mathrm{~ns}$, and with a position resolution at the submicron level. For tests of the FONT5 system the ATF is operated in a mode whereby a train of two or three bunches is extracted from the damping ring and sent down the ATF2 beam line. The bunch separation is determined by the damping ring fill pattern and typically is chosen to be between $140 \mathrm{~ns}$ and either 154 ns (three-bunch mode) or $300 \mathrm{~ns}$ (two-bunch mode).

The FONT5 BPM system (Fig. 3) consists of three stripline BPMs, each of which is instrumented with an analogue processor, and a custom multichannel digitizer. Stripline BPMs were used due to their inherently fast, broadband response and capability to resolve bunches with the required submicron position resolution. In the FONT5 system at ATF, only the vertical plane of the BPMs is routinely instrumented, as only vertical position stabilization is required from the feedback system. The BPMs are described in Sec. II.

\section{STRIPLINE BPMS}

A stripline BPM typically consists of four strips of conducting material that are placed longitudinally along the interior surface of the cylinder forming the local beam pipe. In the stripline BPM design, as used in the FONT5 system, four $12 \mathrm{~cm}$ strips are arranged as two orthogonal diametrically opposed pairs separated by $23.9 \mathrm{~mm}$, as shown in Fig. 4 [11]. The BPMs are installed in the beam line so that the stripline pairs lie in the vertical and horizontal planes. A right-handed coordinate system is used in which the beam is defined to propagate in the $z$ direction, and $y$ to point vertically upwards. Each BPM is mounted on movers (Fig. 5) [12] that permit independent translation along the $x$ and $y$ axes; this allows convenient centering of the beam inside each BPM aperture. The range of motion in both axes is $\pm 1.5 \mathrm{~mm}$ and the minimum step size is $1 \mu \mathrm{m}$.

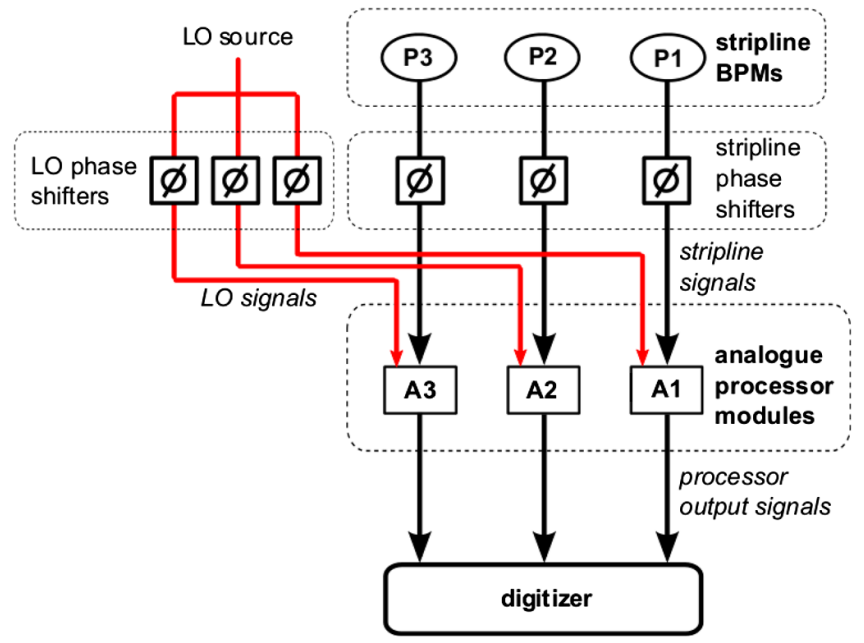

FIG. 3. Schematic of the FONT5 BPM system. For each BPM, a phase shifter is used on one of the stripline signals to adjust the relative path lengths of the two input signals at the BPM processor, and another phase shifter is used to adjust the phase of the LO signal at each processor (see Sec. V).

\section{A. Stripline signals}

When a bunch of charged particles passes through the aperture of the BPM, voltage pulses are induced on each stripline; more details are given in [13]. The voltage pulse is determined by the beam current $I(t)$, the BPM inner radius $R$, the transverse position of the bunch within the $\operatorname{BPM}(x, y)$ and the impedance of the measurement electronics $\rho$.

For illustration, a BPM voltage output was measured by connecting a single stripline directly to an oscilloscope and recording signals due to the successive passage of single electron bunches. An example is given in Fig. 6(a), which shows a characteristic bipolar signal (note that the signal is broadened due to the limited bandwidth of the oscilloscope). The delay of the second pulse relative to the first corresponds to twice the signal propagation time along the stripline, $\sim 2 L / c$, where $L$ is the stripline length. The Fourier transform of this signal is shown in Fig. 6(b). The power spectrum peaks close to $625 \mathrm{MHz}$, which is the frequency corresponding to half the interpulse time delay for $L=12 \mathrm{~cm}$.

\section{B. Signal processing}

For a beam traveling parallel to the BPM axis and with the top and bottom striplines designated as $T$ and $B$ respectively, considering only the vertical offset $y$ (and assuming that $y \ll R$ ), the expressions for the voltages induced on the striplines are [13]

$$
\begin{aligned}
& V_{T}(t) \propto\left(1+\frac{2 y}{R}\right) \rho I(t), \\
& V_{B}(t) \propto\left(1-\frac{2 y}{R}\right) \rho I(t) .
\end{aligned}
$$



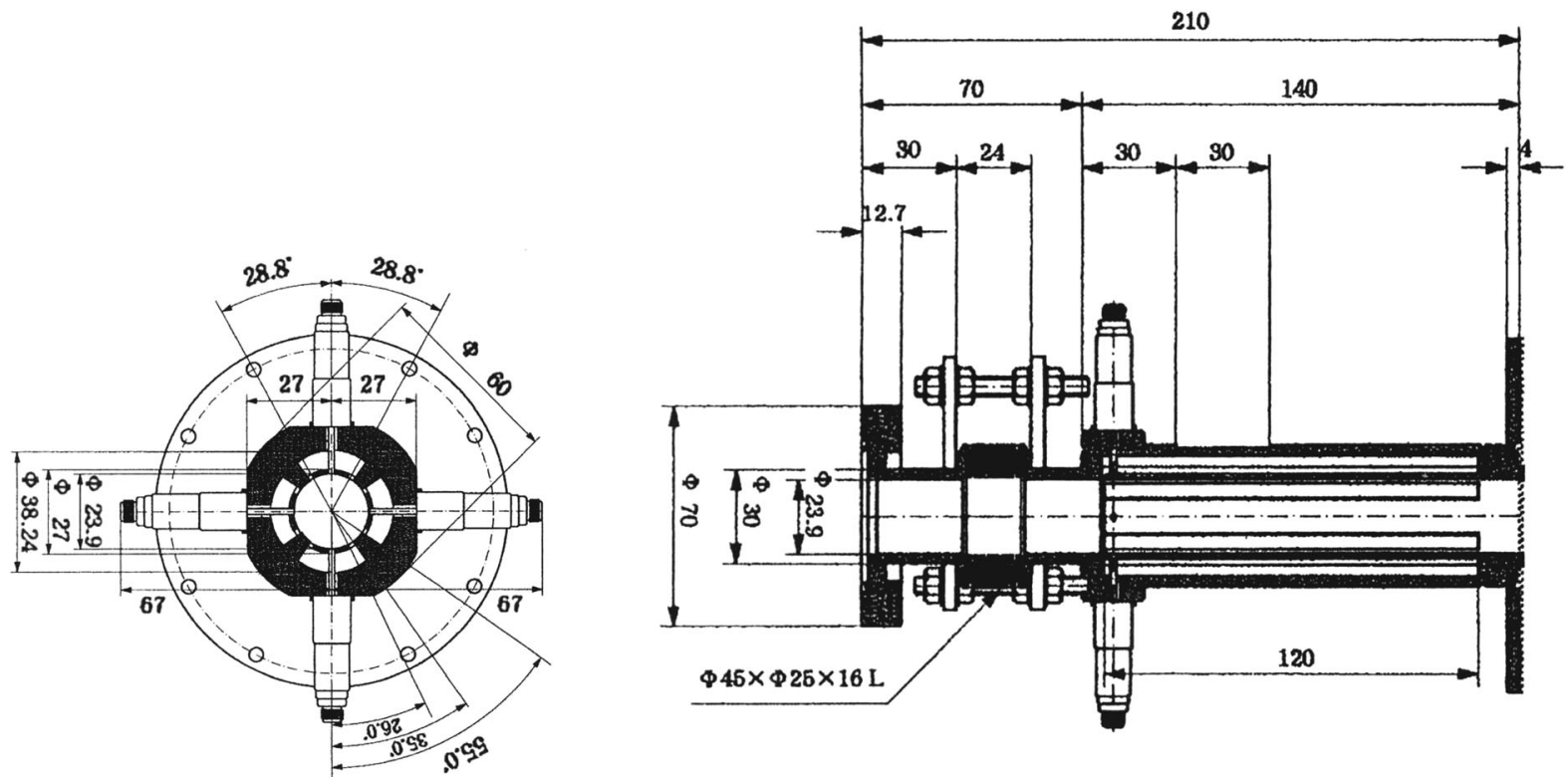

FIG. 4. Technical drawing of the stripline BPMs used as part of the FONT system; lengths are given in mm.

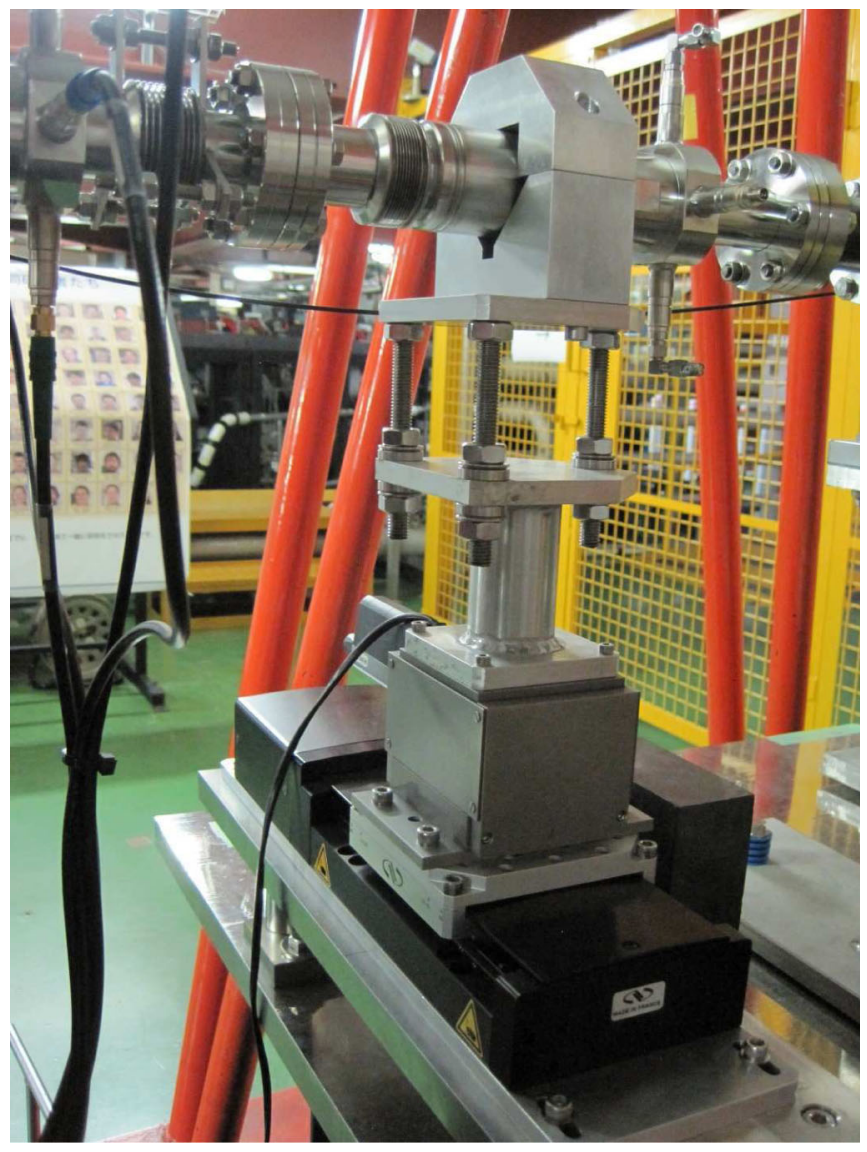

FIG. 5. Photograph of FONT5 stripline BPM P3 and its mover in the ATF2 beam line.
In the difference over sum signal processing scheme used here this pair of signals are added to form the sum $\left(V_{\Sigma}\right)$ and subtracted to form the difference $\left(V_{\Delta}\right)$ :

$$
\begin{gathered}
V_{\Sigma} \equiv g_{\Sigma}\left(V_{T}+V_{B}\right) \propto 2 g_{\Sigma} \rho I(t), \\
V_{\Delta} \equiv g_{\Delta}\left(V_{T}-V_{B}\right) \propto 4 g_{\Delta} \frac{y}{R} \rho I(t),
\end{gathered}
$$

where $g_{\Sigma}$ and $g_{\Delta}$ are the respective gain factors introduced by the signal processing. Note that the $V_{\Delta}$ signal is proportional to the beam position, charge and BPM radius whilst $V_{\Sigma}$ depends only on the charge. Dividing $V_{\Delta}$ by $V_{\Sigma}$ thus yields a quantity that is proportional to the beam position and independent of the bunch charge:
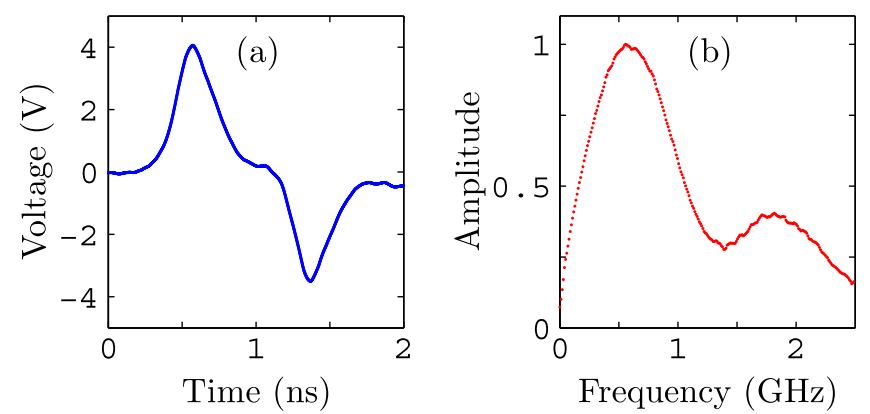

FIG. 6. (a) Stripline output voltage vs time recorded with an oscilloscope (with interpolation). (b) Smoothed Fourier transform of stripline output voltage vs frequency. 


$$
\frac{V_{\Delta}}{V_{\Sigma}}=\frac{2}{R}\left(\frac{g_{\Delta}}{g_{\Sigma}}\right) y \equiv k y
$$

where $k$ is defined to be the "monitor constant" of the BPM and processor system.

\section{ANALOGUE PROCESSORS}

The FONT5 analogue processors (Fig. 7) were designed and constructed at the University of Oxford; further details can be found in [14] and references therein. A photograph of a processor is shown in Fig. 8. The function of the analogue processors is to deliver the $V_{\Delta}$ [Eq. (4)] and $V_{\Sigma}$ [Eq. (3)] signals in a form that can be easily recorded by the digitizer for calculation of the position-dependent, beam charge-independent ratio of the two [Eq. (5)]. Ten processors were built and are used in beam operations at ATF2. A single BPM processor can be used to process the beam position data in either the horizontal or vertical plane; from here on only the vertical plane is considered.

\section{A. Processor design}

The inputs to each processor (Fig. 7) are the stripline voltage signals $V_{T}$ and $V_{B}$ and a $714 \mathrm{MHz}$ local oscillator (LO) signal $V_{\mathrm{LO}}$; the outputs are a down-mixed $V_{\Delta}$ signal along with two down-mixed versions of the sum, $V_{\Sigma}$ and $V_{\Sigma_{Q}}$, which differ in that the LO signal input to the $\Sigma_{Q}$ mixer has a relative phase of $90^{\circ}$. The $714 \mathrm{MHz}$ LO signal is sourced from the ATF damping ring rf system so as to provide a signal that is in principle phase locked to the arrival of the beam at the BPMs. Nett amplification ensures an LO signal amplitude close to the design value (measured at the input to the processor) of $14 \mathrm{dBm}$.

The operation of the processor is as follows: $V_{T}$ and $V_{B}$ are each passed through a low-pass filter with a $1 \mathrm{GHz}$ cutoff frequency [15]. Their sum and difference are then formed by a resistive coupler network and a $180^{\circ}$ hybrid [16], respectively, before both are passed through bandpass filters with a central frequency of approximately 700 and $200 \mathrm{MHz}$ bandwidth. The sum signal is attenuated by the resistive coupler, so that, by design, the gain in the difference channel, $g_{\Delta}$, is a factor $\sim 20$ larger than the gain in the sum channel, $g_{\Sigma}$. This ensures that the sum signal at the mixer input remains at a comfortable level below saturation for the expected beam intensities at ATF, and hence optimizes the position sensitivity and determines the range in linear response for the system.

The difference signal is then used as the RF input to the $\Delta$-channel mixer [17] while the sum signal is split to provide the RF inputs to both the $\Sigma$-channel and the $\Sigma_{Q}$-channel mixers. The LO inputs to the three mixers are obtained by first splitting the primary LO input to the processor in two: one output of this split drives the $\Delta$ channel mixer while the other is input to a $90^{\circ}$ hybrid [18]. The output of this hybrid is a pair of signals in quadrature phase with respect to each other which are used as the LO inputs for the $\Sigma$-channel and $\Sigma_{Q}$-channel mixers. The $\Delta$-channel LO signal is attenuated so that its amplitude matches that of the $\Sigma$-channel and $\Sigma_{Q}$-channel LO signals. A delay cable in the sum channel path is used to match the phase of the RF inputs to the $\Delta$-channel and $\Sigma$-channel mixers. The final stages of processing are identical for the $V_{\Delta}, V_{\Sigma}$ and $V_{\Sigma_{Q}}$ signals. After being mixed with its respective LO signal, each signal is passed through a low-pass filter with a $100 \mathrm{MHz}$ cutoff frequency to yield an output at baseband.

A model of the processor was developed to aid understanding and is documented in [14]. For illustration, example $V_{\Delta}$ and $V_{\Sigma}$ signals, recorded with beam, are shown in Fig. 9, along with the corresponding model predictions using the recorded real BPM stripline signals as inputs. The model reproduces the features of the processed signals. Each baseband signal is then amplified by a nett $17 \mathrm{~dB}$, so as to match the input range of the digitizer, and then passed through a low-pass filter with a $120 \mathrm{MHz}$ cutoff frequency [19] in order to remove any high frequency noise

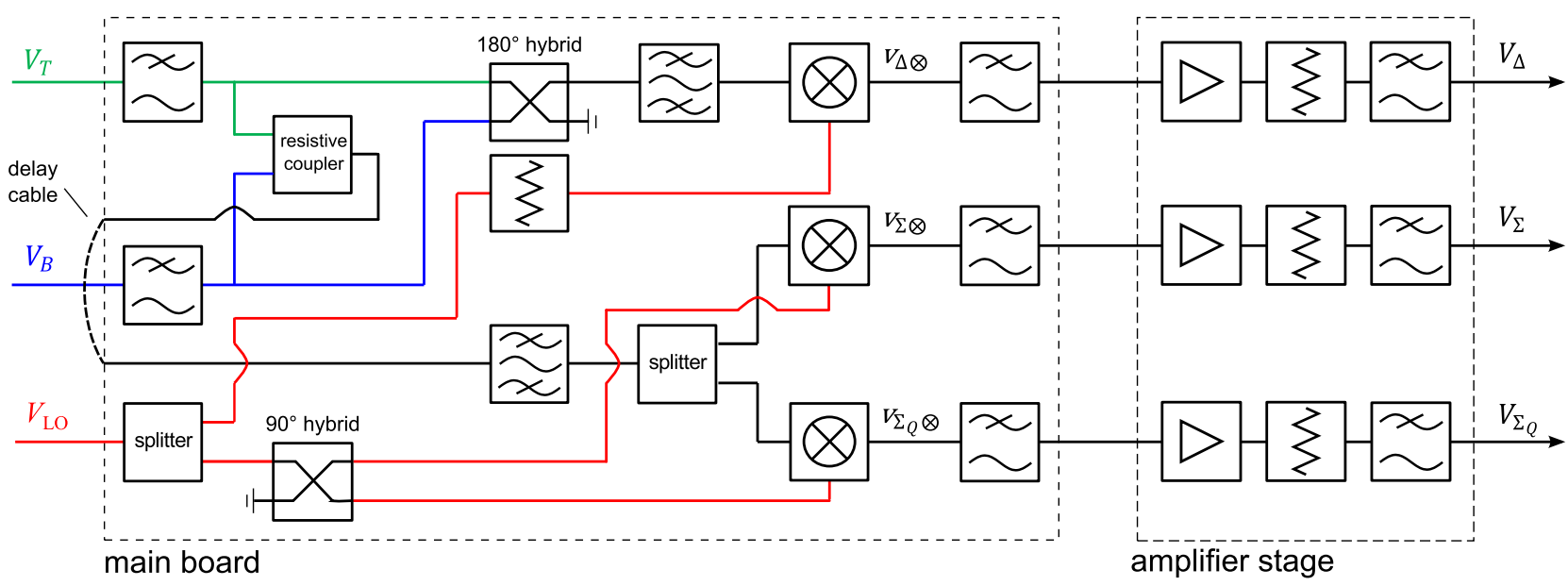

FIG. 7. Schematic diagram illustrating the structure of the FONT5 analogue processor. 


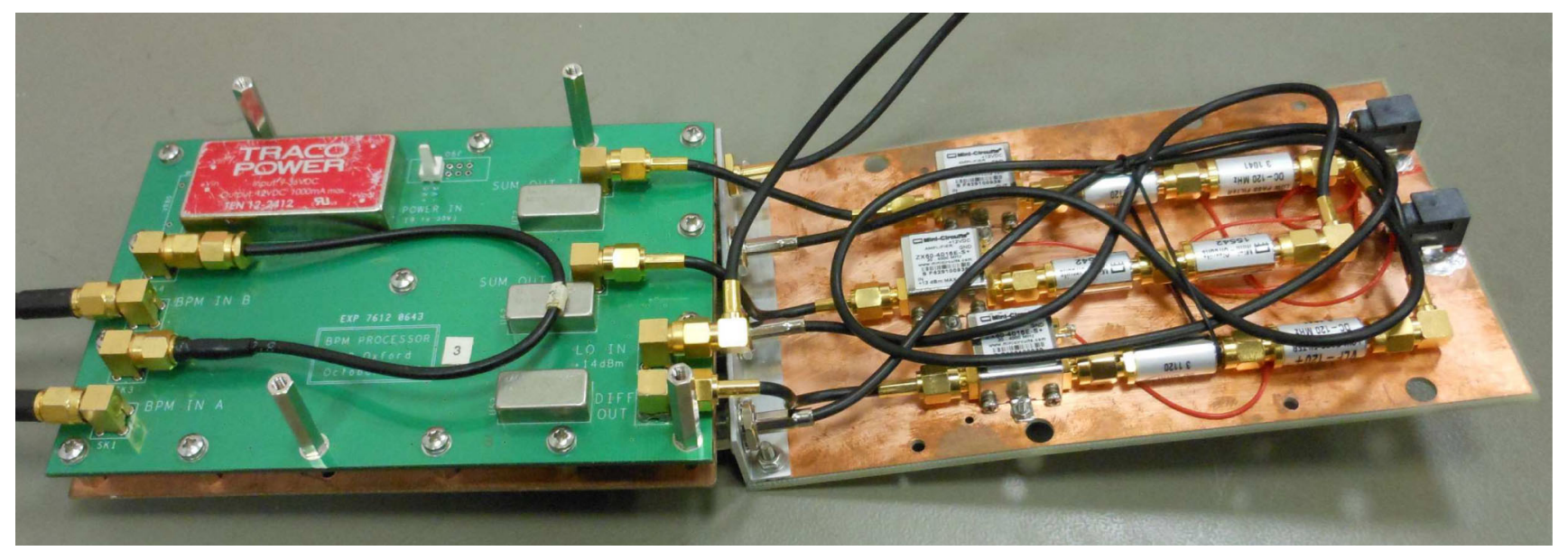

FIG. 8. Photograph of a FONT5 analogue processor. The unit is partially disassembled for viewing purposes.

introduced by the amplifier stage. The processor noise is dominated by the amplifier and low-pass filter, which has a noise bandwidth of $200 \mathrm{MHz}$, and is expected to be $\sim 90 \mu \mathrm{V}$. This would correspond to a noise limit of $\sim 100 \mathrm{~nm}$ on the position resolution, at a bunch charge of $\sim 1 \mathrm{nC}$.

\section{B. Processor latency}

The latency of the processor is defined to be the time interval between the arrival of the $V_{T}$ and $V_{B}$ signals at the inputs and the appearance of the $V_{\Delta}, V_{\Sigma}$ and $V_{\Sigma_{Q}}$ signals
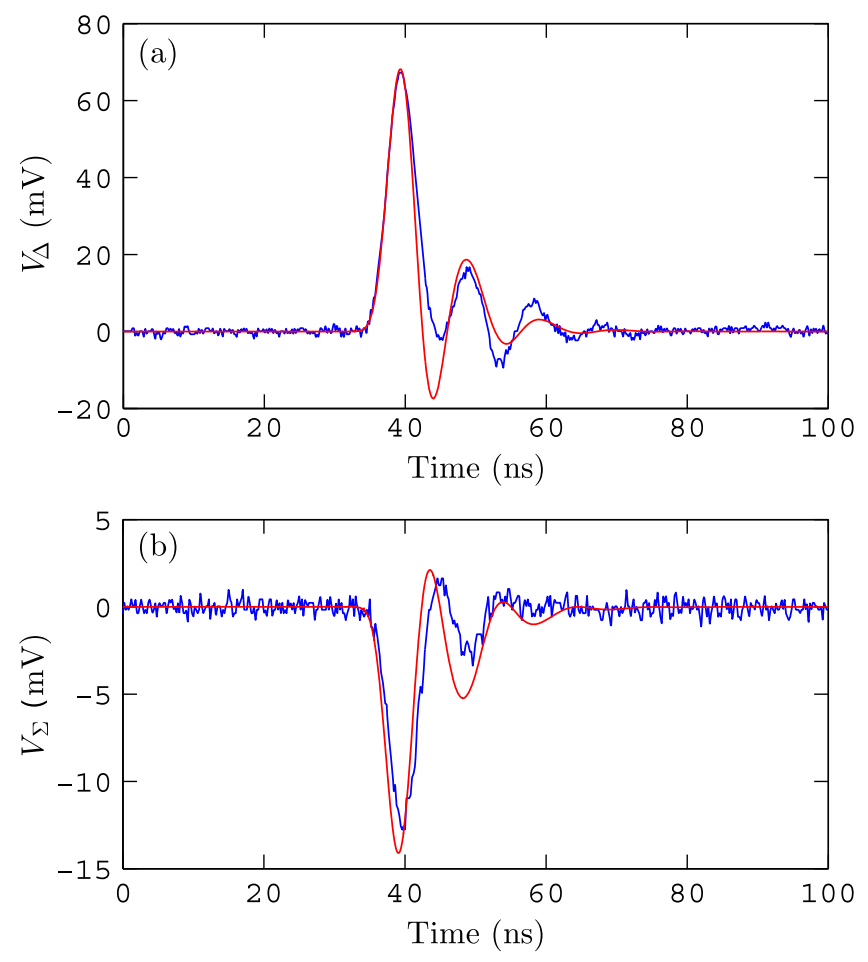

FIG. 9. Recorded (a) $V_{\Delta}$ and (b) $V_{\Sigma}$ waveforms from the processor (blue) compared with the model (red). at the outputs. One of the principal design goals was that the latency should be low, while providing baseband output pulses that are amenable to convenient digitization (Sec. IV). The latency was measured by using a test bench to provide realistic beam-proxy input signals and observing on an oscilloscope the arrival time of the processed $V_{\Delta}$ output. Subtracting from this the time of arrival at the oscilloscope of the $V_{T}$ input when the processor is bypassed, the processor latency before the amplifier stage was found to be $10.4 \pm 0.1 \mathrm{~ns}$, and $15.6 \pm 0.1 \mathrm{~ns}$ including the amplifier stage (Fig. 10).

\section{Mathematical treatment of processor operation}

As any signal can be represented using Fourier analysis as a sum of trigonometric functions, consider a single angular frequency component of the stripline signals $V_{T}$ and $V_{B}$, denoted $v_{T}(\omega)$ and $v_{B}(\omega)$ respectively:

$$
v_{T}(\omega)=v_{T} \sin (\omega t)
$$

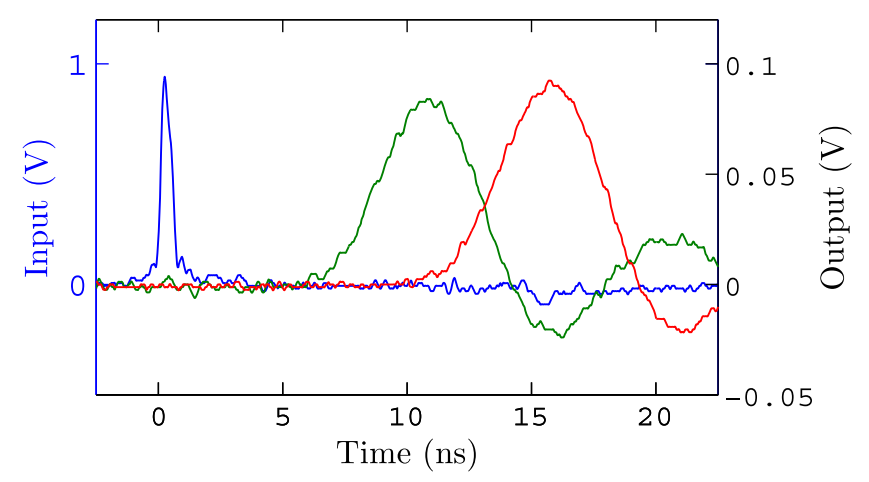

FIG. 10. Input beam proxy $V_{T}$ signal (blue, left-hand scale) and processor output $V_{\Delta}$ signal before the amplifier stage (green, right-hand scale, with factor 5 multiplication), and after the amplifier stage (red, right-hand scale), vs time (ns). The amplifier stage delays the output signal by an additional $5.2 \mathrm{~ns}$. 


$$
\begin{aligned}
v_{B}(\omega) & =v_{B} \sin (\omega t+\theta) \\
& \equiv v_{B}[\cos \theta \sin (\omega t)+\sin \theta \cos (\omega t)],
\end{aligned}
$$

where $v_{T}$ and $v_{B}$ are the amplitudes of frequency $\omega$ in the Fourier decompositions of $V_{T}$ and $V_{B}$ respectively and $\theta$ is the phase offset of $v_{B}(\omega)$ relative to $v_{T}(\omega)$.

An ideal resistive coupler adds $v_{T}(\omega)$ and $v_{B}(\omega)$ to give $v_{\text {coupler }}(\omega)$ and an ideal $180^{\circ}$ hybrid first shifts the phase of $v_{B}(\omega)$ by $180^{\circ}$ and then adds this result to $v_{T}(\omega)$ to form $v_{\text {hybrid }}(\omega)$ :

$$
\begin{aligned}
v_{\text {coupler }}(\omega)= & {\left[v_{T}+v_{B} \cos \theta\right] \sin (\omega t) } \\
& +v_{B} \sin \theta \cos (\omega t), \\
v_{\text {hybrid }}(\omega)= & {\left[v_{T}-v_{B} \cos \theta\right] \sin (\omega t) } \\
& -v_{B} \sin \theta \cos (\omega t) .
\end{aligned}
$$

For each BPM, the two cables connecting the stripline pickoffs to the processor inputs were carefully designed so as to equalize the signal path lengths to within approximately $200 \mu \mathrm{m}$ [20], hence the simplifying assumption $\theta \ll 1$ can be used. For the case where the beam travels close to the center of the BPM, $v_{T} \sim v_{B}$ and the $\cos (\omega t)$ term in the expression for $v_{\text {coupler }}(\omega)$ can be neglected:

$$
\begin{gathered}
v_{\text {coupler }}(\omega) \sim\left[v_{T}+v_{B}\right] \sin (\omega t), \\
v_{\text {hybrid }}(\omega) \sim\left[v_{T}-v_{B}\right] \sin (\omega t)-v_{B} \theta \cos (\omega t) .
\end{gathered}
$$

These signals then travel to the mixers, whose LO signal input has the form

$$
V_{\mathrm{LO}}\left(\omega_{\mathrm{LO}}\right)=V_{\mathrm{LO}} \sin \left(\omega_{\mathrm{LO}} t+\phi_{\mathrm{LO}}\right),
$$

where $V_{\mathrm{LO}}$ is the amplitude of the LO signal, $\omega_{\mathrm{LO}}$ is the angular frequency, and $\phi_{\mathrm{LO}}$ is the phase offset between the LO signal and the RF input signal. Since a $90^{\circ}$ phase shift is applied to the LO signal used for the $\Sigma_{Q}$ mixer, the outputs of the three mixers (Fig. 7) are given by

$$
\begin{array}{r}
v_{\Delta \otimes}(\omega)=v_{\text {hybrid }}(\omega) \cdot V_{\mathrm{LO}} \sin \left(\omega_{\mathrm{LO}} t+\phi_{\mathrm{LO}}\right), \\
v_{\Sigma \otimes}(\omega)=v_{\text {coupler }}(\omega) \cdot V_{\mathrm{LO}} \sin \left(\omega_{\mathrm{LO}} t+\phi_{\mathrm{LO}}\right), \\
v_{\Sigma_{Q} \otimes}(\omega)=v_{\text {coupler }}(\omega) \cdot V_{\mathrm{LO}} \cos \left(\omega_{\mathrm{LO}} t+\phi_{\mathrm{LO}}\right) .
\end{array}
$$

The bandpass filters on the $v_{\text {coupler }}(\omega)$ and $v_{\text {hybrid }}(\omega)$ signals ensure that only a narrow band of frequency components survive to the inputs of the mixers and so $\omega \sim \omega_{\text {LO }}$. The low-pass filters suppress the up-mixed terms in the mixer outputs and so the processor module outputs at baseband (Fig. 7) may be expressed as

$$
\begin{gathered}
V_{\Delta} \propto\left[v_{T}-v_{B}\right] \cos \left(\phi_{\mathrm{LO}}\right)-v_{B} \theta \sin \left(\phi_{\mathrm{LO}}\right), \\
V_{\Sigma} \propto\left[v_{T}+v_{B}\right] \cos \left(\phi_{\mathrm{LO}}\right), \\
V_{\Sigma_{Q}} \propto\left[v_{T}+v_{B}\right] \sin \left(\phi_{\mathrm{LO}}\right),
\end{gathered}
$$

where $v_{T} \equiv v_{T}\left(\omega_{\mathrm{LO}}\right)$ and $v_{B} \equiv v_{B}\left(\omega_{\mathrm{LO}}\right)$. For each BPM the phase $\phi_{\mathrm{LO}}$ can be adjusted using a dedicated LO phase shifter (Fig. 3). For illustration, Fig. 11 shows measured $V_{\Delta}, V_{\Sigma}$ and $V_{\Sigma_{Q}}$ signals for one BPM as $\phi_{\mathrm{LO}}$ is varied through the maximum range of $180^{\circ}$; these exhibit the expected trigonometric functional dependences on $\phi_{\mathrm{LO}}$ [Eqs. (16)-(18)]. It is clear that $V_{\Sigma}$ is maximized when $\phi_{\mathrm{LO}} \sim 0$, which is equivalent to $V_{\Sigma_{Q}} \sim 0$.

Combining the derived expressions of Eqs. (16) and (17) gives the result:

$$
\frac{V_{\Delta}}{V_{\Sigma}} \propto \frac{v_{T}-v_{B}}{v_{T}+v_{B}}-\frac{v_{B}}{v_{T}+v_{B}} \theta \tan \phi_{\mathrm{LO}}
$$

and from Eqs. (17) and (18):

$$
\frac{V_{\Sigma_{Q}}}{V_{\Sigma}}=\tan \phi_{\mathrm{LO}}
$$

The first term in Eq. (19) is directly proportional to the beam position [see Eq. (5)]. For regular beam operations the system is set up (Sec. V) so that, for each BPM, $\phi_{\mathrm{LO}} \sim 0$ and the second term is in general small, in which case the residual phase deviation from zero, $\Delta \phi_{\mathrm{LO}}$, is given by

$$
\Delta \phi_{\mathrm{LO}}=\frac{V_{\Sigma_{Q}}}{V_{\Sigma}} .
$$

For illustration, Fig. 12 shows the measured $V_{\Delta} / V_{\Sigma}$ and $V_{\Sigma_{Q}} / V_{\Sigma}$ signal ratios for one BPM as $\phi_{\mathrm{LO}}$ is varied through

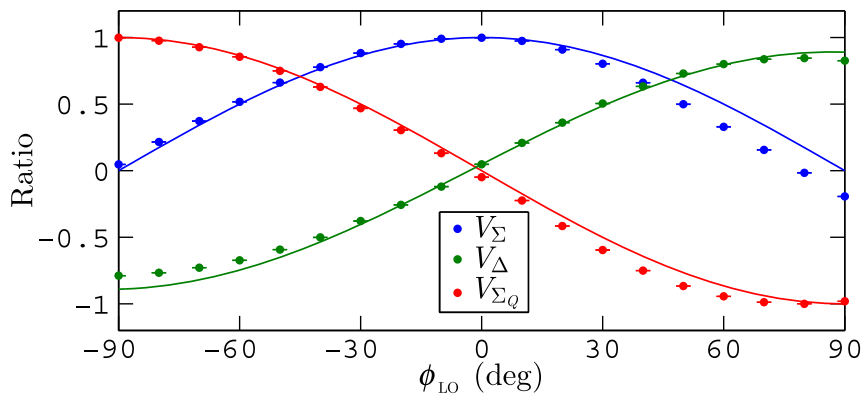

FIG. 11. Illustrative measured $V_{\Delta}, V_{\Sigma}$ and $V_{\Sigma_{Q}}$ signals, vs LO signal phase $\phi_{\mathrm{LO}}$ (degrees of $714 \mathrm{MHz}$ ); each signal has been normalized pulse-by-pulse by $\left(V_{\Sigma}^{2}+V_{\Sigma_{O}}^{2}\right)^{1 / 2}$, which removes the effects of pulse-by-pulse fluctuations in the beam charge. 50 pulses are averaged at each phase setting. Each line shows the respective function in Eqs. (16)-(18); the $V_{\Delta}$ line was normalized to the data point at $\phi_{\mathrm{LO}}=0$. 


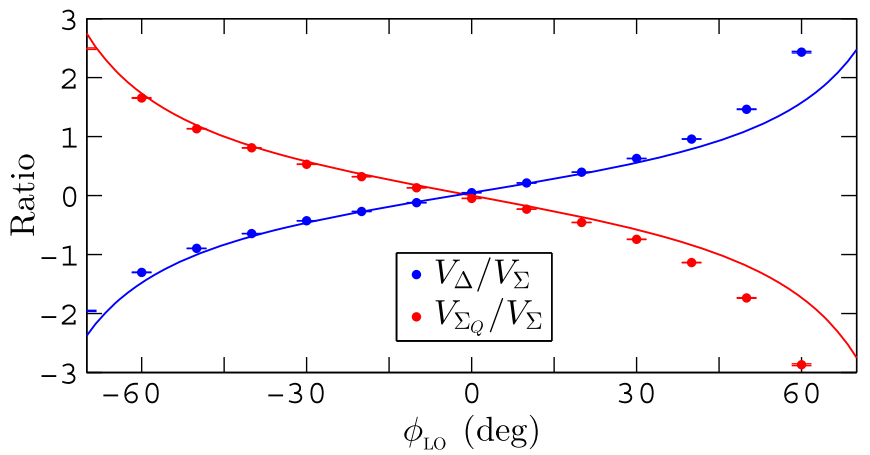

FIG. 12. Illustrative measured ratios $V_{\Delta} / V_{\Sigma}$ and $V_{\Sigma_{Q}} / V_{\Sigma}$ vs LO signal phase $\phi_{\mathrm{LO}}$ (degrees of $714 \mathrm{MHz}$ ); 50 pulses are averaged at each phase setting. The lines show the functions in Eqs. (19) and (20); the $V_{\Delta} / V_{\Sigma}$ prediction was normalized to the data point at $\phi_{\mathrm{LO}}=0$.

the very wide range $\left|\phi_{\mathrm{LO}}\right|<60^{\circ}$; they exhibit the expected dependences on $\phi_{\text {LO }}$ [Eqs. (19) and (20) respectively].

From Eq. (19) it can be seen that the consequence of a static phase offset, $\theta$, between the top and bottom stripline input signals, is the addition to the expected expression for the position of the beam [Eq. (5)] a term ${ }^{1}$ which depends on the residual phase offset $\Delta \phi_{\mathrm{LO}}$ between the $\mathrm{LO}$ and RF inputs to the mixers. If $\Delta \phi_{\mathrm{LO}}$ were constant the quadrature term would appear as a small static offset to the position measurement and would not affect the position resolution.

The LO signal phase stability was measured and is illustrated in Fig. 13. A random variable component, as well as a correlated slower drift (related to the beam line cooling water temperature control), can be seen. The variation from beam pulse to beam pulse is at the level $\left|\Delta \phi_{\mathrm{LO}}\right| \sim 0.5^{\circ}(714 \mathrm{MHz}) \mathrm{rms}[20,21]$. If uncorrected this residual lack of phase stability would cause a noticeable degradation in the position resolution of the BPM system.

Combining Eqs. (5) and (19)-(21),

$$
\frac{V_{\Delta}}{V_{\Sigma}}=k y-k_{\phi} \Delta \phi_{\mathrm{LO}} \equiv y_{\text {raw }}
$$

where $k_{\phi}$ is the parameter that quantifies dependence of the raw beam position, $y_{\text {raw }}$, on $\Delta \phi_{\mathrm{LO}}$, and $k$ is the monitor constant that relates $y_{\text {raw }}$ to the true beam position $y$. Hence the unwanted dependence on $\Delta \phi_{\mathrm{LO}}$ can be corrected in two ways: (i) if $k_{\phi}$ is measured [for example, from the slope around $\left|\Delta \phi_{\mathrm{LO}}\right| \sim 0$ of the $y_{\text {raw }}$ vs $\Delta \phi_{\mathrm{LO}}$ curve (Fig. 12)], the quadrature term in Eq. (22) can be evaluated pulse by pulse and the true position $y$ can be determined by explicitly correcting the raw position:

\footnotetext{
${ }^{1}$ This is sometimes referred to as the quadrature term as it is orthogonal to the desired position term in the expression for $V_{\Delta}$ in Eq. (16).
}

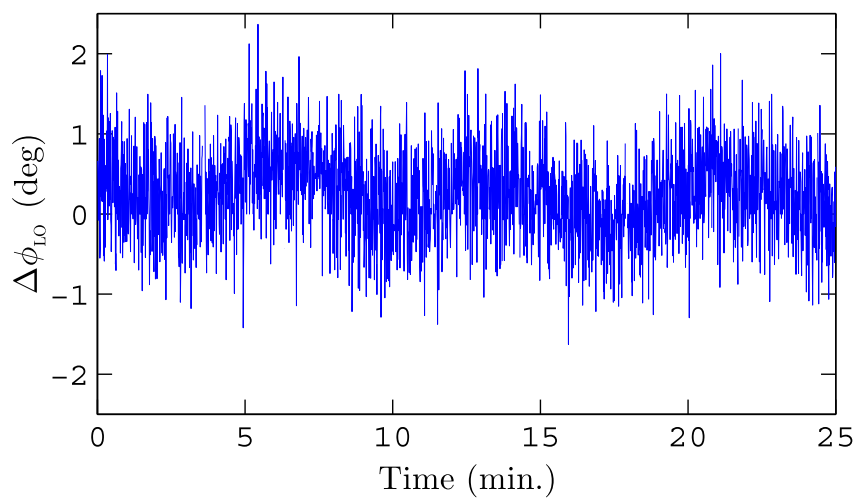

FIG. 13. Illustrative measured LO phase (degrees of $714 \mathrm{MHz}$ ) vs beam extraction time.

$$
y=\frac{1}{k}\left(y_{\text {raw }}+k_{\phi} \Delta \phi_{\mathrm{LO}}\right) .
$$

(ii) $k_{\phi}$ can be minimized by using the stripline phase shifters (Fig. 3) to minimize the static phase offset $\theta$ between the two stripline inputs to the processor.

In recent beam operations method (ii) was used; this is described in Sec. V. Prior to the installation of the stripline phase shifters, method (i) was used.

\section{DIGITIZER}

For each BPM, digitization of the three analogue processor outputs (Fig. 7) is performed in the FONT5 digital feedback board (Fig. 14). The board is built around a Xilinx Virtex-5 field programmable gate array (FPGA) [22] and features nine Texas Instruments ADS5474 14-bit analogue-to-digital converters (ADCs) [23], which are transformer coupled to block the response to low frequency noise, giving an input voltage range of $\pm 0.5 \mathrm{~V}$. As the expected noise level from the BPM processor corresponds

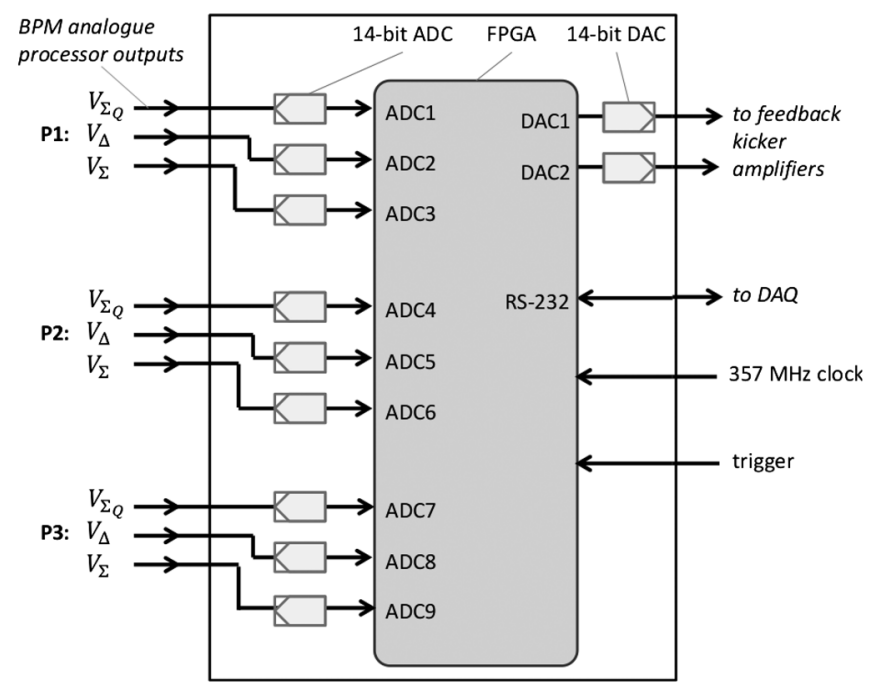

FIG. 14. Schematic of the FONT5 digital feedback board. 
to $\sim 1.5$ counts at 14-bit resolution, only the most significant 13 bits are input to the FPGA for further processing and data acquisition. This reduces congestion of the signals in the FPGA fabric.

Also included are three LTC2624 quad 16-bit digital-toanalogue converters (DACs) [24]; of the twelve available DAC channels, nine are connected directly to the ADCs and used to correct the intrinsic zero offset of each ADC channel. An RS-232 port enables the board to communicate remotely for configuration and data acquisition purposes.

An external trigger signal that precedes the firing of the ATF damping ring beam extraction kicker is input to the board to synchronize data acquisition to the bunch arrival time. The sampling window of the ADCs is a fixed 164 samples in length but an internal delay on the trigger signal allows the start of the window to be set relative to the trigger with a precision of 2.8 ns. Finer control over the sample timing is exercised through manipulation of the ADC clocks.

Each ADC is clocked with a $357 \mathrm{MHz}$ signal derived from the $714 \mathrm{MHz}$ local oscillator signal to ensure sample timing stability on the trigger-to-trigger time scale. A schematic of the ADC clock generation in the FONT5 firmware is shown in Fig. 15 and illustrates how the ADCs on the board are arranged in three groups of three with each group sharing a common clock. The three ADCs of a group are used to sample the $V_{\Delta}, V_{\Sigma}$ and $V_{\Sigma_{Q}}$ outputs of a single BPM processor. The variable delay element on each ADC clock output from the FPGA allows the absolute sample timing to be varied separately for each group in increments of $70 \mathrm{ps}$.

For each BPM the sample timing is set such that the sampled value of the peak of the $V_{\Sigma}$ waveform is at a maximum. Individual control of the ADC clock delays allows optimal sample timing to be achieved for multiple BPMs simultaneously despite the differences in the arrival time of the bunch at each BPM as well as the different lengths of the signal path from each BPM to the FONT5 board.

For each trigger, once sampling is complete the nine digitized waveforms are transmitted over the serial RS-232
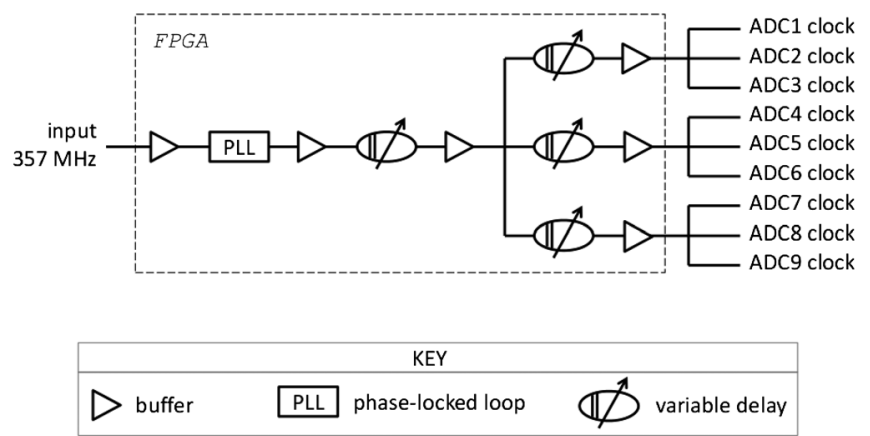

FIG. 15. Schematic of the ADC clock generation in the FONT5 board firmware. data connection. A PC running the custom data acquisition (DAQ) software [25] is used for live monitoring of the beam position and also enables the creation of a permanent record of the sampled data for off-line analysis. Further details of the operation of the FONT5 board are given in [26].

For illustration, typical digitized $V_{\Delta}, V_{\Sigma}$ and $V_{\Sigma_{Q}}$ waveforms from one BPM are shown in Fig. 16; these correspond to measurement of a single-bunch beam of charge approximately $1 \mathrm{nC}$. The $V_{\Sigma}$ waveform for a train of three bunches with an interbunch separation of $154 \mathrm{~ns}$ is
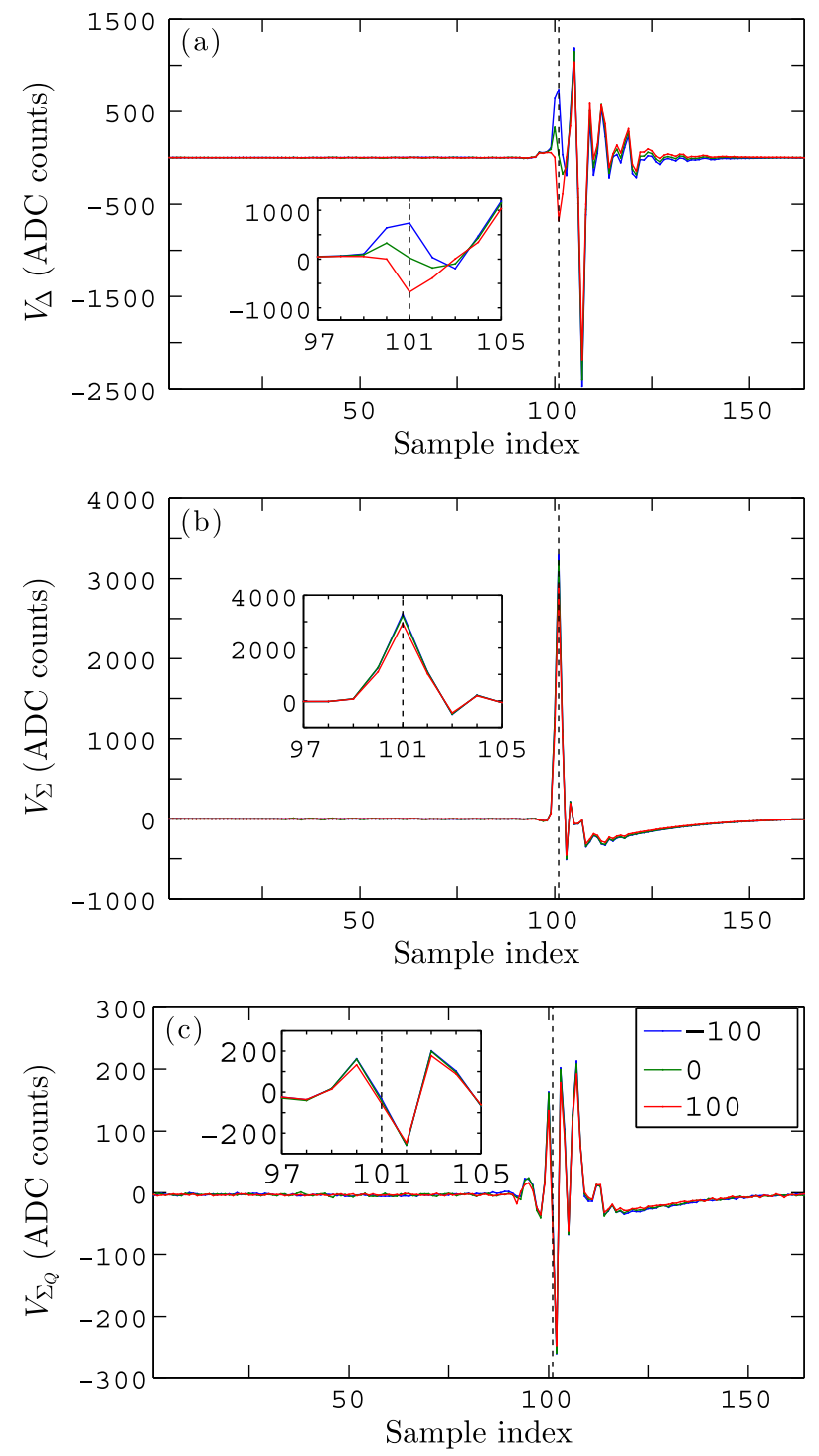

FIG. 16. Example recorded (a) $V_{\Delta}$, (b) $V_{\Sigma}$, and (c) $V_{\Sigma_{Q}}$ waveforms for three illustrative vertical beam position settings: $-100 \mu \mathrm{m}, 0 \mu \mathrm{m}$ and $100 \mu \mathrm{m}$ (set using the BPM vertical mover). The bunch sample, as indicated by the vertical dotted line, is set to correspond to the peak of the $V_{\Sigma}$ signal. A zoom of the region around the bunch sample is shown inset for each signal. Note that the large negative peak six samples after the bunch sample in (a) is due to round-trip reflections through the striplines arriving back at the input ports of the hybrid. 


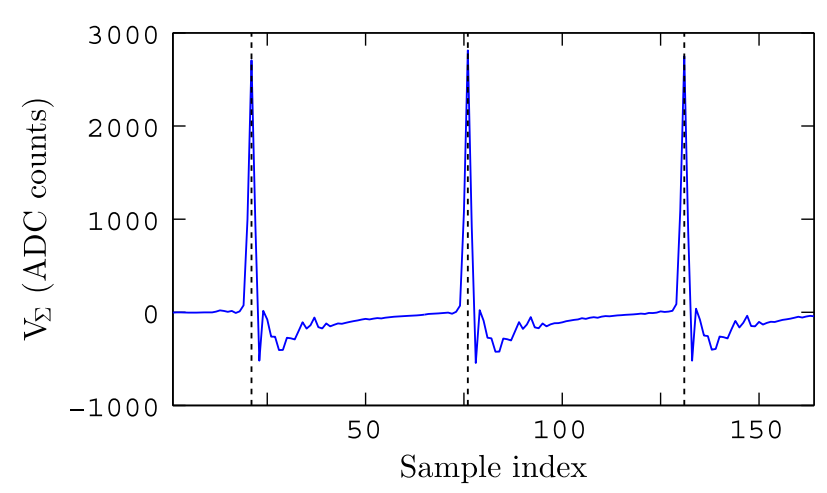

FIG. 17. Example recorded $V_{\Sigma}$ waveform for a train of three bunches with an interbunch separation of 154 ns. The bunch samples are indicated by the vertical dotted lines.

shown in Fig. 17. We refer to the samples corresponding to the peak of the $V_{\Sigma}$ waveform as the "bunch samples"; from here on we discuss, unless stated otherwise, only the bunch samples.

For each BPM the raw bunch position, $y_{\text {raw }}$, is calculated in real time from the $V_{\Delta}$ and $V_{\Sigma}$ bunch samples [Eq. (22)]. This calculation is implemented in the FONT5 board firmware by using a lookup table for the division by $V_{\Sigma}$ in order to minimize the calculation time. The raw bunch position is used for online monitoring purposes, and as the input to a beam-based feedback system, which is described elsewhere [25-30]. Here we report in detail the system performance in terms of its beam position measurement capability.

\section{SYSTEM PREPARATION}

At the start of each data-taking period, for each BPM the LO signal phase $\phi_{\mathrm{LO}}$ is set, the system sensitivity to LO signal phase variation, $\Delta \phi_{\mathrm{LO}}$, is minimized, and the monitor constant $k$ is measured. We describe these procedures briefly below.

\section{A. LO signal phase setting}

For each BPM the maximum signal-to-noise ratio for the processor output signals is obtained when the phases of the LO signals input to the $\Sigma$ and $\Delta$ mixers match the phases of the RF inputs. Since, by design, the phase of the LO signal at the $\Sigma_{Q}$ mixer is $90^{\circ}$ with respect to the RF input, this is achieved by setting the $\mathrm{LO}$ phase shifter on the input to the processor (Fig. 3) so that $\left\langle V_{\Sigma_{Q}}\right\rangle \sim 0$. In practice this is done in an automated fashion using the system control and DAQ program [25].

\section{B. LO signal phase sensitivity minimization}

For each BPM the stripline phase shifter (Fig. 3) is adjusted so as to minimize the static phase offset, $\theta$, between $V_{T}$ and $V_{B}$ [20]. By this means a residual sensitivity of $\left|k_{\phi}\right|<0.1 \mu \mathrm{m} /{ }^{\circ}$ (714 MHz) is obtained.

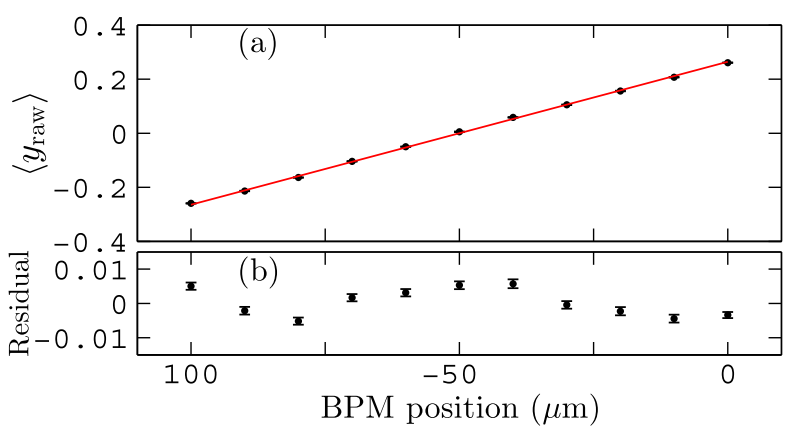

FIG. 18. (a) $y_{\text {raw }}$ vs vertical BPM position. 25 beam pulses are averaged at each setting; the red line is a linear fit to the data. (b) Residual of the data with respect to the fitted line. The maximum deviation of the residuals corresponds to $\sim 2 \mu \mathrm{m}$, and is consistent with periodic beam drift over the course of a few minutes.

This is done in an automated fashion using the system control and DAQ program. Given the measured LO signal phase jitter of $0.5^{\circ}$ one expects this effect to contribute less than $50 \mathrm{~nm}$ to the position resolution.

\section{Position scale calibration}

For each BPM the vertical and horizontal movers are first set to ensure that the beam trajectory passes close to its center in the $x-y$ plane. The system is then calibrated by translating the BPM position in steps with the vertical mover. The monitor constant $k$ [Eq. (22)] is derived by performing a linear fit of $y_{\text {raw }}$ to the set BPM position. An example calibration plot is shown in Fig. 18. The value of $k$ is typically measured to be around $2.5 \times 10^{-3} \mu \mathrm{m}^{-1}$, which is consistent with the ratio of $g_{\Delta}$ and $g_{\Sigma}$ [Eq. (5)] derived from bench measurements of the BPM processor response to controlled beam-proxy input signals [14]. This value varies slightly from BPM processor to processor due to variation in component nominal values and actual build. It also depends on the settings of $\phi_{\mathrm{LO}}$ and the bunch sample timing (see e.g., Fig. 16), but the system preparation is designed to minimize sensitivity to these settings. For illustration an ensemble of $k$ values for a given BPM

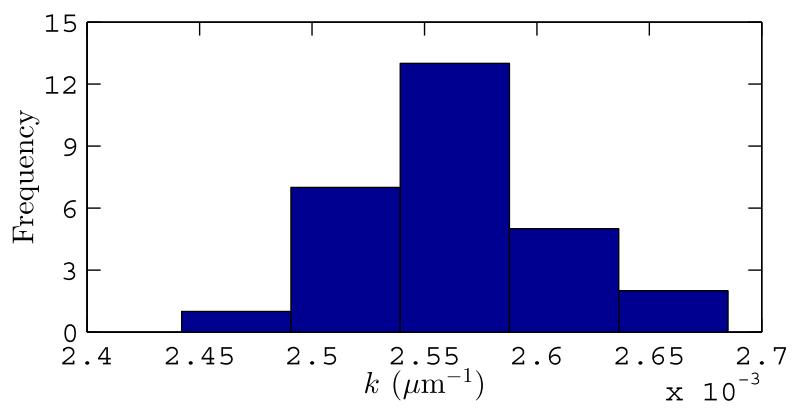

FIG. 19. Illustrative distribution of measured values of $k$ for a given processor connected to a given BPM, recorded over a 20-month period. 
processor connected to a given BPM is shown in Fig. 19; the measurements were taken over a 20 -month period and the rms variation is $1.9 \%$ of the mean.

\section{PERFORMANCE}

The performance of the BPM system is described in terms of its range of linear response and resolution for position measurements.

\section{A. Range of linear response}

This is defined to be the range over which the system responds linearly to a change in beam position. A nonlinear response is expected if the input signal to a mixer is large enough to cause its output to saturate. Saturation will be avoided if the mixer RF input signal level is small compared with the design LO input signal level, $\sim 7 \mathrm{dBm}$. For optimum resolution, the stripline BPM signals can be attenuated to ensure that for the nominal beam charge $(\sim 1 \mathrm{nC})$ the $\Sigma$-channel signal level is comfortably below the mixer saturation point. The processor output is then expected to be linear as long as $\left|V_{\Delta} / V_{\Sigma}\right| \lesssim 1$. From Eq. (22), and noting that $k \sim 2.5 \times 10^{-3} \mu \mathrm{m}^{-1}$, a linear response is expected for $|y| \lesssim 400 \mu \mathrm{m}$.

As an illustration, Fig. 20 shows the raw beam position measured in BPM P3 as a function of the vertical BPM position. The measurements visibly deviate from linearity for beam positions more than $500 \mu \mathrm{m}$ from the electrical zero point; within this range, the deviation from linearity is less than $2 \%$. A larger range could be obtained with the system described here by increasing the attenuation of the stripline signals input to the processor, although this would degrade the position resolution. A future design with higher level mixers could achieve, using presently available components, an increase in the dynamic range by a factor 2 , without penalty to the resolution.

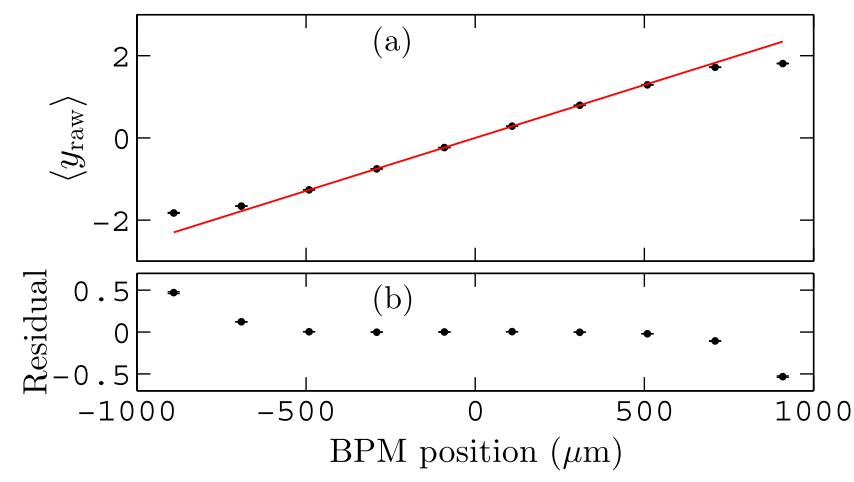

FIG. 20. (a) $y_{\text {raw }}$ vs vertical BPM position. 50 beam pulses are averaged at each setting; the red line is a linear fit to the central four data points. (b) Residual of the data with respect to the fitted line. The maximum deviation of the residuals corresponds to $\sim 200 \mu \mathrm{m}$ at the extremal points, clearly showing the effect of saturation in the mixers when operated outside of their linear range.

\section{B. Position resolution}

The resolution of the system is determined by comparing the beam position measured in one BPM with the position predicted at that BPM on the basis of the beam positions measured in the other two BPMs. For illustration, such a set of beam position measurements is shown in Fig. 21.

The predicted position at BPM $\mathrm{P} i$ is given by

$$
y_{i}^{\text {pred }}=C_{i j} y_{j}^{\text {meas }}+C_{i k} y_{k}^{\text {meas }}
$$

where $y_{j}^{\text {meas }}$ and $y_{k}^{\text {meas }}$ are the measured positions in BPMs $\mathrm{P} j$ and $\mathrm{P} k$ respectively, and $C_{i j}$ and $C_{i k}$ are prediction coefficients. For a given beam pulse, the position residual for BPM $\mathrm{P} i$ is defined by

$$
\delta y_{i} \equiv y_{i}^{\text {meas }}-y_{i}^{\text {pred }}
$$

and the distribution of position residuals measured on successive beam pulses can be characterized by the Gaussian function:

$$
\frac{d N}{d\left(\delta_{y_{i}}\right)}=A_{i} e^{-\left(\delta y_{i}\right)^{2} / 2 \sigma_{i}^{2}}
$$

of width $\sigma_{i}$, where $A_{i}$ is a normalization factor. Assuming that the three BPMs have the same resolution, $\sigma$,

$$
\sigma \equiv \frac{\sigma_{i}}{\sqrt{1+C_{i j}^{2}+C_{i k}^{2}}} .
$$

The prediction coefficients, $C_{i j}$ and $C_{i k}$, are derived using the MAD8 model [31] of the beam line lattice to evaluate the beam transfer matrix elements between locations $i$ and $j$, and $i$ and $k$, respectively. Position residuals, normalized by the prediction coefficients as in Eq. (27), are shown in Fig. 21. These residuals yield a resolution estimate, for a centered beam with a bunch charge of approximately $1 \mathrm{nC}$, of

$$
\sigma=291 \pm 10 \mathrm{~nm}
$$

which is world leading in terms of the position resolution obtained in stripline BPMs in single-pass beam mode. Given the system preparation described in Sec. V such a level of performance is achieved routinely in beam operations.

For comparison, a global least-squares fit can be performed to explicitly minimize $\sigma$. This method assumes no a priori knowledge of the model of beam transport between the three BPMs [32,33]. For the same data set presented in Fig. 21, this yields a value for the resolution of $262 \pm 11 \mathrm{~nm}$. As this method removes any correlated components of the BPM position data, and also allows for variation in the individual BPM scale factors, this result represents the minimum possible resolution that could, in 

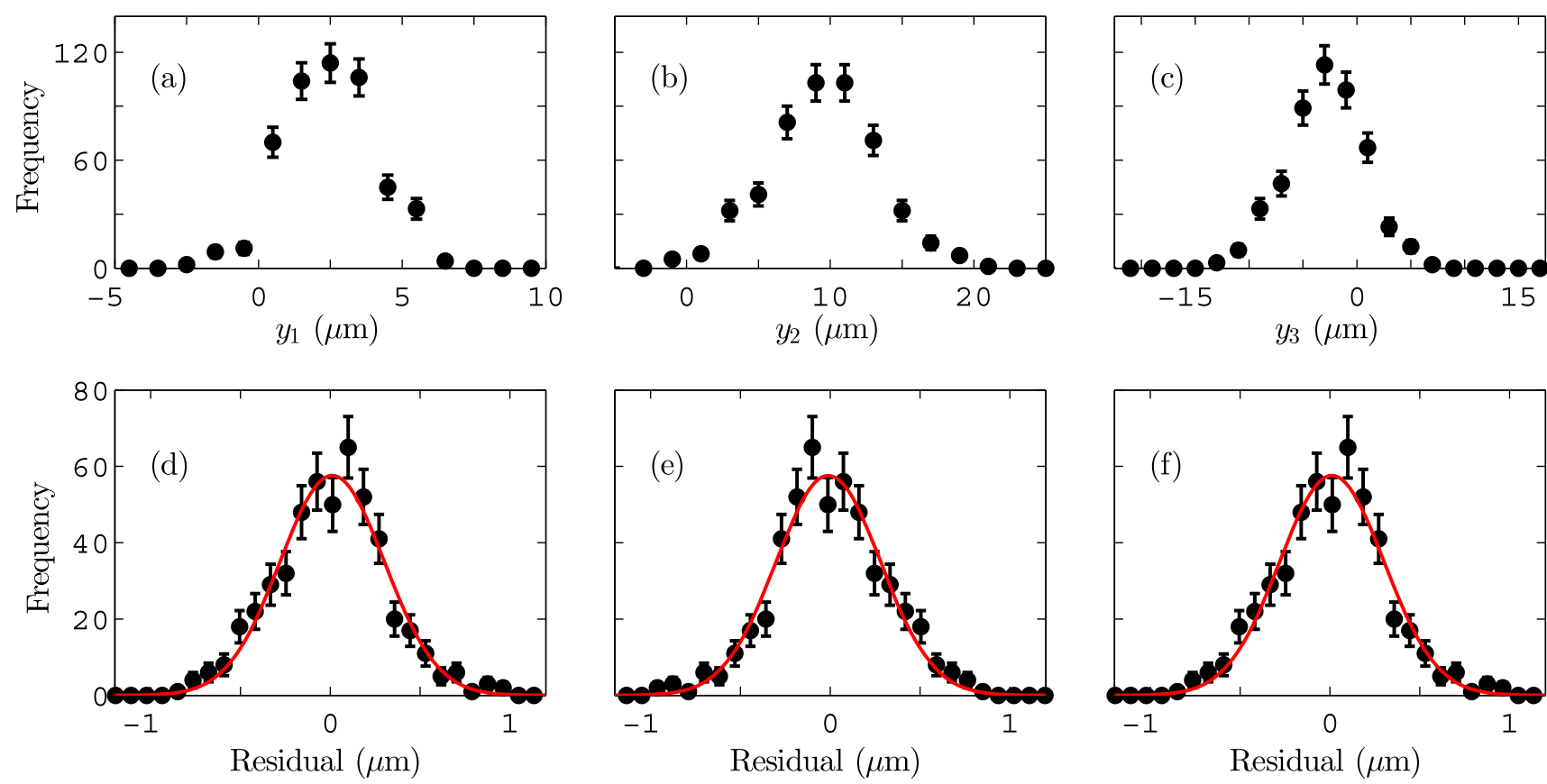

FIG. 21. Top row: distribution of the beam position measured in BPM (a) P1, (b) P2 and (c) P3 for a set of 500 consecutive beam pulses. Bottom row: distributions of the corresponding position residuals for BPMs (d) P1, (e) P2 and (f) P3; the red lines show Gaussian fits to the respective data.

principle, be attained, if for example any residual correlated effects were accounted for. In contrast, the value obtained using the beam line model better represents the actual minimum sensitivity attainable in a given position measurement. The value attained with the least-squares fit is consistent with that expected from the measured system noise. For example, for $\mathrm{P} 3$ the noise on $V_{\Delta}$, in the absence of beam, was 1.5 counts and this, combined with the observation that $\left\langle V_{\Sigma}\right\rangle \sim 2300$ counts, suggests a contribution of $\sim 260 \mathrm{~nm}$ to the apparent position jitter.

\section{Resolution dependence on charge and position}

From Eq. (23), neglecting the $k_{\phi}$ term one expects the position resolution $\sigma$ to be determined by the noise on the $V_{\Delta}$ and $V_{\Sigma}$ signals $\left(\sigma_{V_{\Delta}}\right.$ and $\sigma_{V_{\Sigma}}$ respectively):

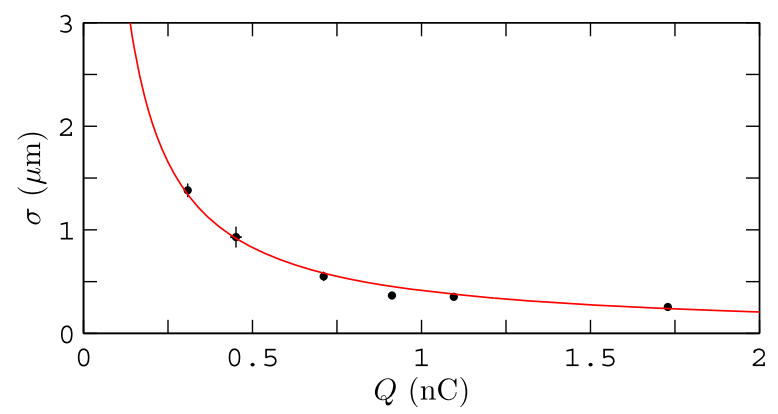

FIG. 22. Position resolution vs beam charge; the red line shows a fit of the function $\sigma=p / Q$, where $p$ is a parameter. Note that for this data set the raw stripline signals were attenuated by $6 \mathrm{~dB}$ in order to handle the very large signal levels at the higher charge settings, which would otherwise saturate the mixer.

$$
\begin{aligned}
\sigma^{2} & =\frac{1}{k^{2} V_{\Sigma}^{2}}\left(\sigma_{V_{\Delta}}\right)^{2}+\frac{V_{\Delta}^{2}}{k^{2} V_{\Sigma}^{4}}\left(\sigma_{V_{\Sigma}}\right)^{2} \\
& \simeq \frac{\sigma_{V_{\Delta}}^{2}}{k^{2} V_{\Sigma}^{2}}\left(1+k^{2} y^{2}\right)
\end{aligned}
$$

for a similar magnitude noise in the sum and difference channels. For constant position offset, $y$, the resolution is expected to vary inversely with $V_{\Sigma}$, i.e., inversely with the beam charge. Figure 22 shows the resolution for a data run that includes different beam charges. The charge dependence is as expected. This data was taken with $6 \mathrm{~dB}$ of stripline attenuation; comparable resolution could be achieved at half the charge with this attenuation removed.

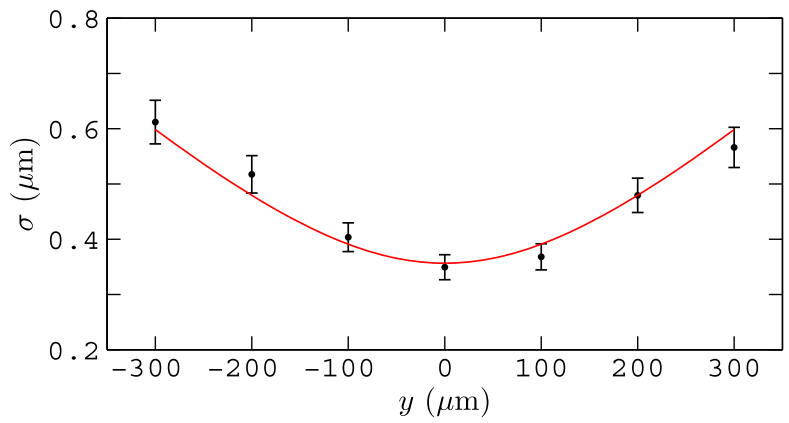

FIG. 23. Position resolution vs beam position; the red line shows a fit of the function $\sigma=p_{1}\left(y^{2}+p_{2}\right)^{1 / 2}$, where $p_{1}$ and $p_{2}$ are parameters. 
For fixed beam charge, the resolution is also expected to vary with $V_{\Delta}$, i.e., with beam position. It is expected to vary approximately linearly at large position offsets, and quadratically for roughly centered beam, as given by Eq. (28). Figure 23 shows the resolution for a series of sequential data runs obtained with different beam positions. For this data set the BPM movers were used to produce an equal apparent beam offset in all three BPMs. The position dependence is as expected and the data shows that the resolution degrades by approximately $1 \mathrm{~nm}$ per micron of beam offset, for large beam offsets.

\section{CONCLUSIONS}

The design and performance of a stripline BPM system based on an analogue signal processing scheme has been presented. The system was designed for low-latency operation and high resolution measurement of beam position. The latency of the analogue processing electronics was measured to be $15.6 \pm 0.1 \mathrm{~ns}$. The position resolution was measured to be $291 \pm 10 \mathrm{~nm}$ for a beam of bunch charge approximately $1 \mathrm{nC}$. At this charge a linear range of $|y|<500 \mu \mathrm{m}$ is observed, giving a dynamic range of $\sim 60 \mathrm{~dB}$. The resolution was found to scale with bunch charge and beam position in the expected manner. The measured performance is close to that expected from the measured system noise, and within a factor 3 of the theoretical noise limit of $\sim 100 \mathrm{~nm}$. For an ideal optimized implementation of this architecture, a factor 2 to 3 improvement in the resolution could, in principle, be achieved.

\section{ACKNOWLEDGMENTS}

We thank the KEK ATF staff for their outstanding logistical support and providing the beam-time and the necessary stable operating conditions for this research. In addition we thank our ATF2 collaborators for their help and support; in particular the University of Valencia IFIC group for providing the BPM mover system. We acknowledge financial support for this research from the UK Science and Technology Facilities Council via the John Adams Institute, University of Oxford, and CERN, CLIC-UK Collaboration, Contract No. K E1869/DG/CLIC. The research leading to these results has received funding from the European Commission under the FP7 Research Infrastructures project EuCARD, Grant agreement No. 227579.

[1] C. Adolphsen et al., The ILC technical design report, volume 3: Accelerator, Technical Report No. JAI-2013001, 2013.

[2] M. Aicheler, P. Burrows, M. Draper, T. Garvey, P. Lebrun, K. Peach, N. Phinney, H. Schmickler, D. Schulte, and N. Toge, A multi-TeV linear collider based on CLIC technology: CLIC conceptual design report, Technical Report No. CERN-2012-007, 2012.

[3] M. Altarelli et al., The European X-Ray Free-Electron Laser: Technical design report, DESY Technical Report No. 2006-097, 2006.

[4] http://www-pnp.physics.ox.ac.uk/ font/index.html.

[5] F. Hinode, S. Kawabata, H. Matsumoto, K. Oide, K. Takata, S. Takeda, and J. Urawaka, Accelerator Test Facility: Design and study report, KEK Technical Report No. 95-4, 1995.

[6] B. Grishanov et al., ATF2 proposal vol. 1, KEK Technical Report No. 2005-2, 2005.

[7] P. Raimondi and A. Seryi, Novel Final Focus Design for Future Linear Colliders, Phys. Rev. Lett. 86, 3779 (2001).

[8] G. R. White et al., Experimental Validation of a Novel Compact Focusing Scheme for Future Energy-Frontier Linear Lepton Colliders, Phys. Rev. Lett. 112, 034802 (2014).

[9] P. Bambade et al., Present status and first results of the final focus beam line at the KEK Accelerator Test Facility, Phys. Rev. ST Accel. Beams 13, 042801 (2010).

[10] R. J. Apsimon, D. R. Bett, P. N. Burrows, G. B. Christian, B. Constance, M. R. Davis, A. Gerbershagen, C. Perry, and J. Resta-Lopez, The FONT5 bunch-by-bunch position and angle feedback system at ATF2, Phys. Procedia 37, 2063 (2012).

[11] N. Terunuma (private communication).

[12] A. Faus-Golfe (private communication).

[13] R. T. Avery, A. Faltens, and E. Hartwig, Non-intercepting monitor for beam current and position, IEEE Trans. Nucl. Sci. 18, 920 (1971).

[14] R. J. Apsimon, The development and implementation of a beam position monitoring system for use in the FONT feedback system at ATF2, D. Phil. thesis, University of Oxford, 2011.

[15] http://www.minicircuits.com/pdfs/LFCN-1000.pdf.

[16] http://www.minicircuits.com/pdfs/SCPJ-2-9.pdf.

[17] http://www.minicircuits.com/pdfs/SYM-2.pdf.

[18] http://www.minicircuits.com/pdfs/HPQ-08.pdf.

[19] http://www.minicircuits.com/pdfs/VLF-120.pdf.

[20] M. R. Davis, The development of intra-train beam stabilisation system prototypes for a future linear collider, D. Phil. thesis, University of Oxford, 2014.

[21] P. N. Burrows, D. R. Bett, N. Blaskovic Kraljevic, G. B. Christian, M. R. Davis, C. Perry, R. J. Apsimon, B. Constance, A. Gerbershagen, and J. Resta López, Development of a turn-by-turn beam position monitoring system for multiple bunch operations of the ATF damping ring, in Proceedings of the 3rd International Particle Accelerator Conference, New Orleans, LA, 2012 (IEEE, Piscataway, NJ, 2012), pp. 930-932.

[22] http://www.xilinx.com/support/documentation/data_sheets/ ds100.pdf.

[23] http://www.ti.com/lit/ds/symlink/ads5474.pdf.

[24] cds.linear.com/docs/en/datasheet/2604fd.pdf.

[25] D. R. Bett, The development of a fast intra-train beambased feedback system capable of operating on the bunch trains of the International Linear Collider, D.Phil. thesis, University of Oxford, 2013. 
[26] B. D. Constance, Design and beam testing of a fast, digital intra-train feedback system and its potential for application at the International Linear Collider, D.Phil. thesis, University of Oxford, 2011.

[27] G. B. Christian, D. R. Bett, N. Blaskovic Kraljevic, P. N. Burrows, M. R. Davis, Y. Kim, and C. Perry, A sub-micron resolution, wide-band, stripline BPM system for driving bunch-by-bunch feed-back and feed-forward systems at ATF, in Proceedings of the 5th International Particle Accelerator Conference, Dresden, Germany, 2014 (JACoW, Geneva, Switzerland, 2014), pp. 13581360.

[28] M. R. Davis, D. R. Bett, P. N. Burrows, N. Blaskovic Kraljevic, G. B. Christian, Y. I. Kim, and C. Perry, Applications of stripline and cavity beam position monitors in low-latency, high precision, intra-train feedback systems, in Proceedings of the 2nd International Beam Instrumentation Conference, Oxford, UK, 2013 (JACoW, Geneva, Switzerland, 2013), pp. 630-633.

[29] P. N. Burrows, D. R. Bett, N. Blaskovic Kraljevic, G. B. Christian, M. R. Davis, Y. I. Kim, C. Perry, R. J. Apsimon, B. Constance, and A. Gerbershagen, Latest performance results from the FONT5 intra train beam position feedback system at ATF, in Proceedings of the 4th International Particle Accelerator Conference, IPAC2013, Shanghai, China, 2013 (JACoW, Shanghai, China, 2013), pp. 3049-3051.

[30] D. R. Bett, N. Blaskovic Kraljevic, P. N. Burrows, G. B. Christian, M. R. Davis, C. Perry, R. J. Apsimon, B. Constance, A. Gerbershagen, and J. Resta Lopez, Latest performance results from the FONT5 intra-train beam position and angle feedback system at ATF2, in Proceedings of the 3rd International Particle Accelerator Conference, New Orleans, LA, 2012 (Ref. [21]), pp. 2864-2866.

[31] http://mad.web.cern.ch/mad/.

[32] N. Baboi, J. LundNielsen, D. Noelle, W. Riesch, T. Traber, J. Kruse, and M. Wendt, Resolution studies at beam position monitors at the FLASH facility at DESY, AIP Conf. Proc. 868, 227 (2006).

[33] T. Suwada, N. Kamikubota, H. Fukuma, N. Akasaka, and H. Kobayashi, Stripline-type beam-position-monitor system for single-bunch electron/positron beams, Nucl. Instrum. Methods Phys. Res., Sect. A 440, 307 (2000). 